\title{
AN EXPLORATORY STUDY OF NOMOPHOBIA IN POST GRADUATE RESIDENTS OF A TEACHING HOSPITAL IN CENTRAL INDIA
}

\author{
DR. PRITAM CHANDAK \\ MBBS M.D (Psychiatry) \\ M.APA (USA) M.EPA (EUROPE) M. ACAMH (UK) M.IPS \\ Fellowship (Child \& Adolescent Psychiatry), London \\ Diploma (Child play therapy), London \\ Clinical fellow (Child \& Adolescent Psychiatry), NIMHANS
}

\section{Background:}

The extensive use of mobile phones and its addiction brings in newer psychological problems like nomophobia which necessitates close examination of its impacts on people's health. Despite growing concerns over the increasing incidence of nomophobia there is paucity of research on the various factors affecting it.

Aim:

This study aims at assessing the prevalence of nomophobia and its relationship with various clinical and socio-demographic factors.

\section{Materials \& Methods:}

100 post graduate residents were included in the study after informed consent and ethics committee approval. Self reported semistructured questionnaire which included details about sociodemographic profile, questions pertaining to factors affecting nomophobia and Nomophobia Questionnaire (NMP-Q) were administered. Data was analyzed statistically

\section{Results:}

Nomophobia was seen in $38 \%$ of the participants with significantly more prevalence in younger age group and in females. Majority of the participants were using mobile phone since $5-10$ years $(71 \%)$. Around $46 \%$ participants used mobile phone for 2-3 hours per day with most of them(47\%) checking their phones 25-50 times per day. More than half of the participants (59\%) spent 500-1000 rupees per month as their mobile expenses. Physical symptoms due to mobile phone use were reported by $37 \%$ of the participants and they were seen significantly more in those with nomophobia.

\section{Conclusion:}

This study not only gives useful insight into nomophobia and its risk factors, but it is also one of the few studies to explore the relationship between clinical and socio-demographic factors related to nomophobia.

\begin{tabular}{|c|c|c|c|c|}
\hline Table 1 & $\begin{array}{c}\text { Socio Demographic } \\
\text { Profile }\end{array}$ & $\begin{array}{c}\text { Nomophob } \\
\text { ia } \\
\text { Present }\end{array}$ & $\begin{array}{c}\text { Nomophob } \\
\text { ia } \\
\text { Absent }\end{array}$ & $\begin{array}{c}\text { Significan } \\
\text { ce }\end{array}$ \\
\hline \multirow[t]{2}{*}{ Age } & $23-25$ years & 27 & 28 & \multirow{2}{*}{$\begin{array}{l}\chi 2=6.38 \\
\mathbf{P}=\mathbf{0 . 0 1}\end{array}$} \\
\hline & $26-28$ years & 11 & 34 & \\
\hline \multirow[t]{2}{*}{ Gender } & Male & 14 & 37 & \multirow{2}{*}{$\begin{array}{l}\chi 2=4.91 \\
\mathbf{P}=\mathbf{0 . 0 2}\end{array}$} \\
\hline & Female & 24 & 25 & \\
\hline \multirow{2}{*}{$\begin{array}{l}\text { Marital } \\
\text { status }\end{array}$} & Single & 36 & 56 & \multirow{2}{*}{$\begin{array}{l}\chi 2=0.62 \\
P=0.42\end{array}$} \\
\hline & Married & 2 & 6 & \\
\hline \multirow{2}{*}{ Residence } & Rural & 15 & 21 & \multirow{2}{*}{$\begin{array}{l}\chi 2=0.32 \\
P=0.57\end{array}$} \\
\hline & Urban & 23 & 41 & \\
\hline \multirow{3}{*}{$\begin{array}{c}\text { Education } \\
\text { year }\end{array}$} & First year residency & 13 & 18 & \multirow{3}{*}{$\begin{array}{c}\chi 2=0.48 \\
P=0.78\end{array}$} \\
\hline & $\begin{array}{r}\text { Second year } \\
\text { residency }\end{array}$ & 15 & 24 & \\
\hline & Third year residency & 10 & 20 & \\
\hline \multirow[t]{4}{*}{ Department } & Preclinical & 10 & 12 & \multirow{4}{*}{$\begin{array}{c}\chi 2=1.35 \\
P=0.71\end{array}$} \\
\hline & Para clinical & 8 & 19 & \\
\hline & Medicine allied & 9 & 14 & \\
\hline & Surgery allied & 11 & 17 & \\
\hline Total & & 38 & 62 & \\
\hline
\end{tabular}

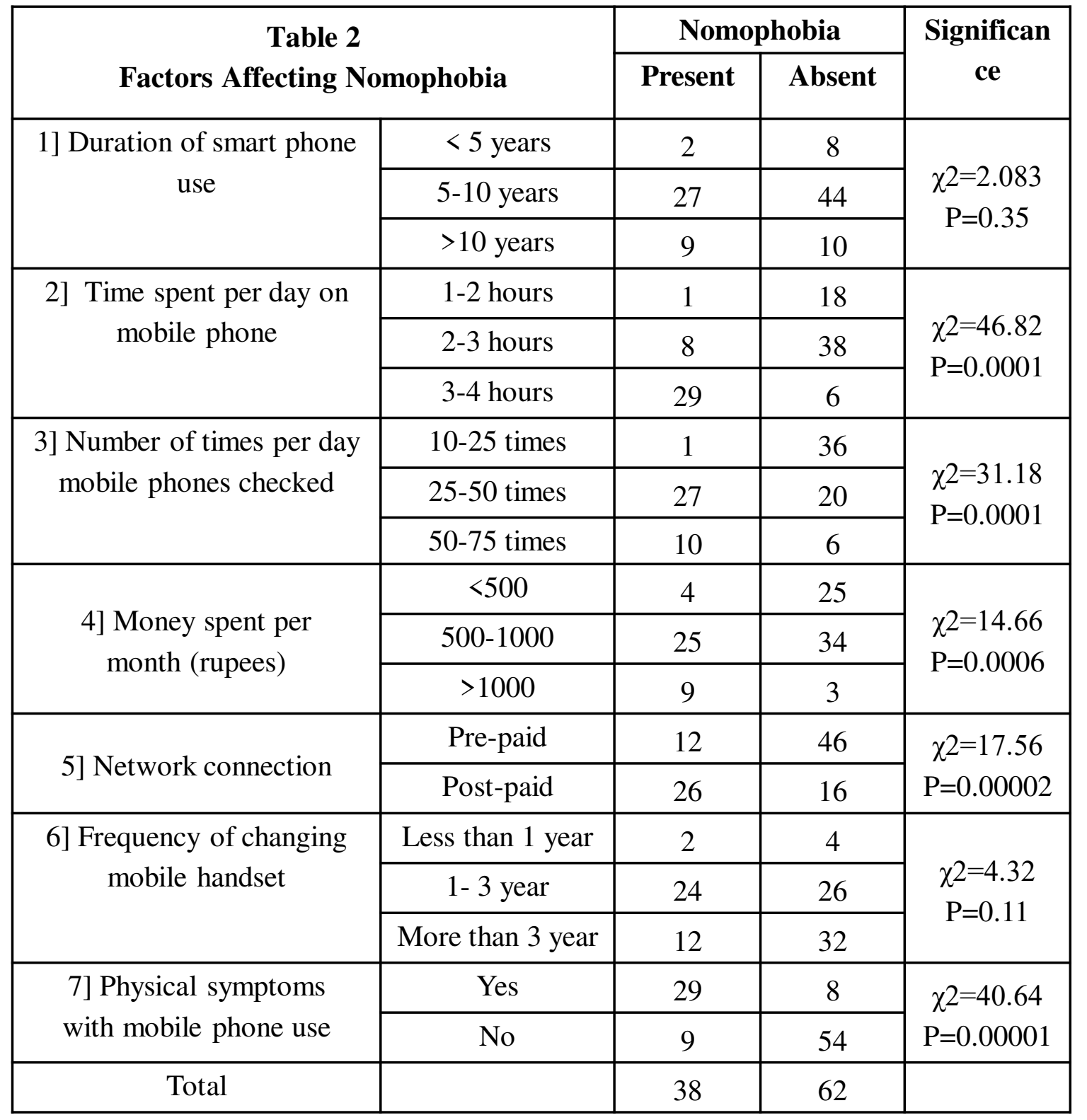

